\title{
Epstein-Barr virus-associated acute disseminated encephalomyelitis successfully treated with rituximab: a case report
}

\author{
Seoyeon $\mathrm{Kim}^{1}$, Seon-Jae $\mathrm{Ahn}^{1,2}$, Kon $\mathrm{Chu}^{1,3}$ \\ ${ }^{1}$ Department of Neurology, Seoul National University Hospital, Seoul, Korea \\ ${ }^{2}$ Center for Hospital Medicine, Seoul National University Hospital, Seoul, Korea \\ ${ }^{3}$ Comprehensive Epilepsy Center, Seoul National University Hospital, Seoul National University College of Medicine, Seoul, Korea
}

Acute disseminated encephalomyelitis (ADEM) is a monophasic central nervous system inflammatory demyelinating disorder clinically defined by multifocal neurologic symptoms with encephalopathy. Brain magnetic resonance imaging most often reveals multiple T2 hyperintense lesions consistent with demyelination. High-dose corticosteroids are the current treatment of choice, and intravenous immunoglobulin or plasmapheresis is considered in steroid-unresponsive cases. The majority of patients show favorable outcomes with full clinical recovery and complete or partial resolution of previous lesions. For patients who are refractory to multiple immunomodulatory agents, other treatments such as rituximab have been used. We present a patient who developed ADEM after Epstein-Barr virus infection who achieved full recovery with immunotherapy including rituximab.

Keywords: Acute disseminated encephalomyelitis, Rituximab, Epstein-Barr virus

\section{Introduction}

Acute disseminated encephalomyelitis (ADEM) is an inflammatory demyelinating disorder of the central nervous system (CNS) characterized by acute polyfocal neurologic symptoms with encephalopathy. In most cases, brain magnetic resonance imaging (MRI) shows abnormalities consistent with demyelination. Typical MRI findings are multiple, hyperintense, poorly-marginated lesions with bilateral asymmetric cerebral involvement in T2-weighted and fluid-attenuated inversion recovery imaging [1]. Tumefactive demyelinating lesions resembling CNS neoplasia or pseudoleukodystrophic patterns have been reported $[2,3]$. Most patients show an excellent response to systemic steroids, and serial MRI usually reveals complete or partial resolution of previous lesions [1]. However, approximately $20 \%$ of patients with ADEM require additional immunotherapy such as intravenous immunoglobulin (IVIg) or plasmapheresis, and these pa- tients are more likely to show poor clinical outcomes [4]. No consensus has been established on how to continue treatment after failure of second-line therapies. We present a case of ADEM in a patient whose clinical symptoms and brain MRI abnormalities completely resolved with third-line rituximab treatment after failing to show improvement with steroid and IVIg administration.

\section{Case Report}

A 42-year-old previously healthy male presented with gradually progressive memory impairment that started 1 month previously. The patient complained of difficulty concentrating at work and recalling recent memories; he could not remember what happened minutes ago. In addition, his personality changed from an introvert to an extrovert along with increased impulsive

Received: April 14, 2021 Revised: April 29, 2021 Accepted: May 3, 2021

Correspondence: Kon Chu

Department of Neurology, Seoul National University Hospital, 101 Daehak-ro, Jongno-gu, Seoul 03080, Korea

E-mail: stemcell.snu@gmail.com

ORCID: https://orcid.org/0000-0001-5863-0302

Copyright (C) 2021 by The Korean Encephalitis and Neuroinflammation Society

This is an open access article distributed under the terms of the Creative Commons Attribution Non-Commercial License (http://creativecommons.org/licenses/by-nc/4.0/) which permits unrestricted non-commercial use, distribution, and reproduction in any medium, provided the original work is properly cited. 
behavior. His past medical history included an upper respiratory infection that lasted longer than 1 month.

Neurologic examination revealed mild dysarthria and left leg weakness of Medical Research Council grade 4+. He scored 18 points on the Mini-Mental State Examination (MMSE). Brain MRI showed diffuse confluent T2 hyperintensity in bilateral cerebral white matter without any diffusion restriction, abnormal enhancement, or cerebral blood volume increase (Figure 1). An analysis of the cerebrospinal fluid (CSF) showed lymphocytic pleocytosis of 139 white blood cells (WBC) $/ \mathrm{mm}^{3}$ (94\% lymphocytes) and an elevated protein level of $123.9 \mathrm{mg} / \mathrm{dL}$. Epstein-Barr virus (EBV) DNA was present in the CSF by polymerase chain reactions (PCR). Whole-body positron emission tomography scan and computed tomography of the chest and abdomen were normal.

The patient received intravenous acyclovir at a dose of $10 \mathrm{mg} / \mathrm{kg}$ for 5 weeks and a high-dose corticosteroid. Slight improvement in memory function was noted, but the cerebral white matter lesions showed no interval change on follow-up MRI. A Follow-up CSF study showed decreased but persisting lymphocytic pleocytosis of $19 \mathrm{WBC} / \mathrm{mm}^{3}$. Follow-up PCR failed to detect EBV DNA in the CSF, and the EBV viral load in whole blood was 1,535 copies/mL. Subsequent IVIg treatment for 5 days did not show additional improvement.

As a third-line immunotherapy, intravenous rituximab (375 mg/ $\mathrm{m}^{2}$ ) was administered at regular intervals (the first four sessions were administered weekly, then monthly). By the end of the fourth cycle, the patient's clinical symptoms recovered to baseline function, with an MMSE score of 30 and no residual left leg weakness. EBV viral load was not detected in whole blood, and CSF analysis showed a decreased WBC count of $9 / \mathrm{mm}^{3}$ with negative EBV DNA. After the seventh cycle of rituximab, the extent of the cerebral white matter involvement started to decrease (Figure 1). Twenty months from symptom onset, after the 11th cycle of rituximab, no residual lesion was seen on brain MRI.

\section{Discussion}

Although the precise etiology of ADEM is unknown, many viruses (measles, herpes, varicella, mumps, EBV, and influenza) have been implicated in its pathogenesis. The clinical diagnosis of ADEM is suggested by a close temporal relationship between an infectious incident and the onset of leukoencephalopathic neurologic symptoms [5]. Current hypotheses involve the cross-reaction of viral antigens with myelin components by molecular mimicry, leading to an inflammatory cascade [6].

Figure 1 Clinical timeline of immunotherapy and corresponding follow-up brain magnetic resonance imaging, CSF cell counts, and MMSE scores

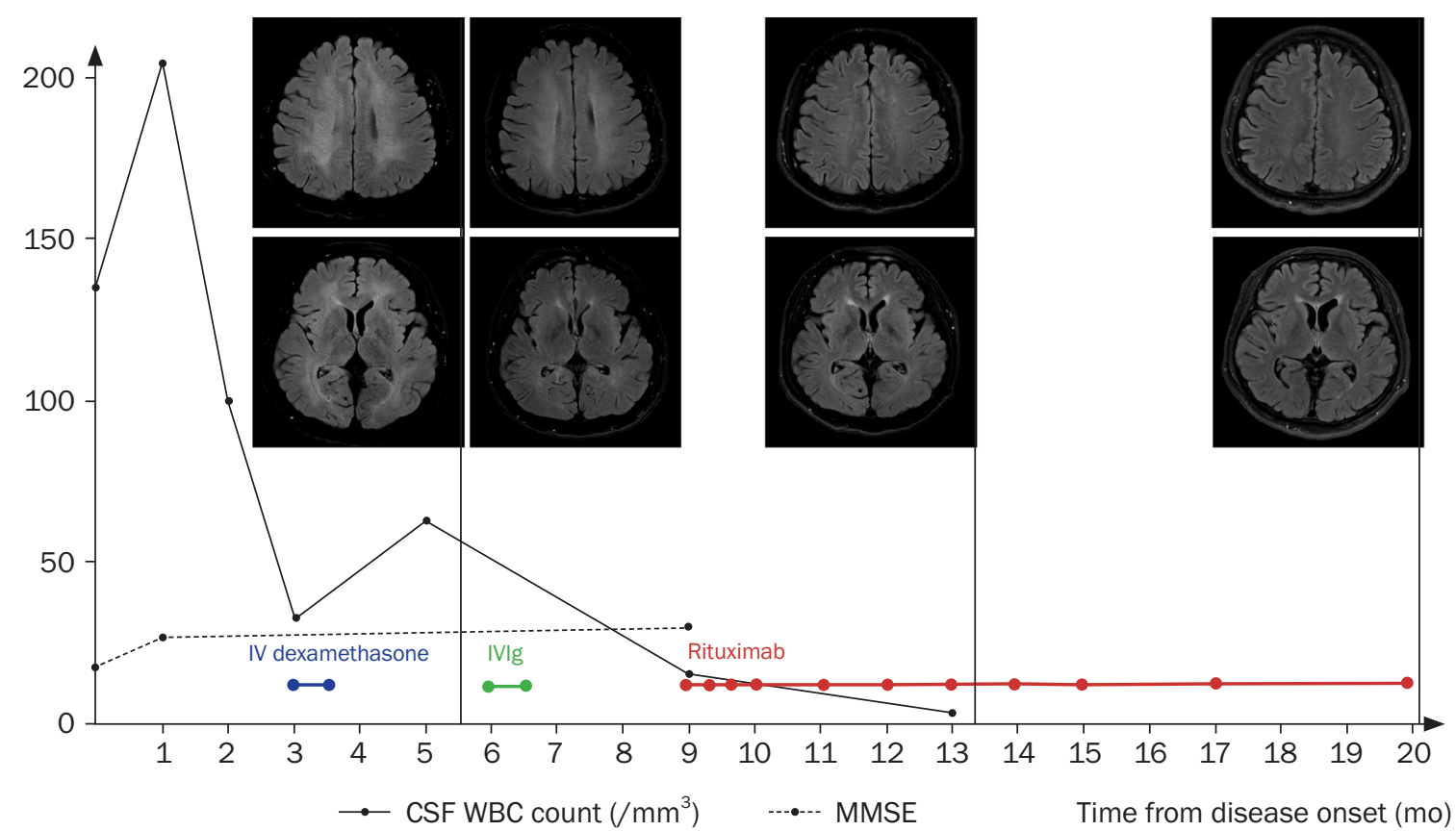

CSF, cerebrospinal fluid; MMSE, Mini-Mental State Examination; IVlg, intravenous immunoglobulin; WBC, white blood cells. 


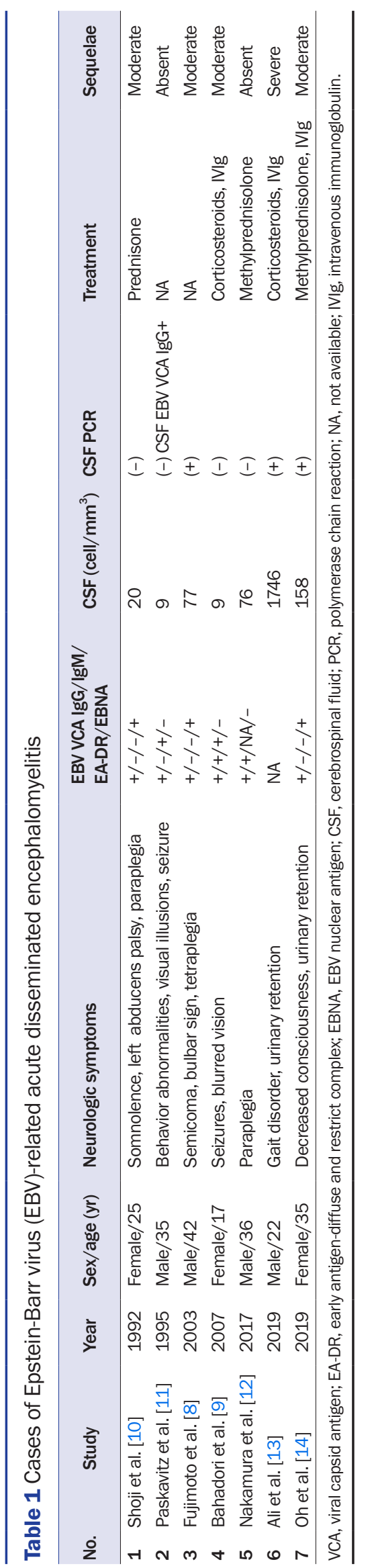

EBV, also known as human herpesvirus 4, primarily targets B cells via interaction of the viral envelope glycoprotein gp350 and CD21/C3d expressed on B cells [7]. EBV infection is mostly asymptomatic in healthy individuals, but a small number can develop CNS complications including demyelinating disease, acute encephalitis, acute cerebellar ataxia, meningitis, or myelitis [8]. A few cases of EBV infection-related ADEM have been reported (Table 1 [8-14]).

The pathogenesis of EBV-associated neurologic disease can be immune-mediated, infectious, or both [9]. In our case, considering that the CSF lymphocytic pleocytosis persisted after treatment with antiviral agents and negative conversion of EBV DNA, postinfectious ADEM secondary to EBV infection is the most likely explanation. The predilection of EBV for B cells might explain why rituximab, an anti-CD20 monoclonal antibody targeting B cells, resulted in marked clinical improvement.

The current treatment approach for ADEM consists of early immunotherapy, but no definitive protocol exists due to a lack of randomized studies. Based on expert opinions and observational studies, high-dose corticosteroids are accepted widely as firstline therapy. In steroid-unresponsive cases, use of IVIg or plasmapheresis has been reported in a small number of publications. Despite common use in multiple sclerosis (MS), rituximab rarely has been administered to patients with ADEM, and the few previous studies available concentrated on a heterogenous group of myelin oligodendrocyte glycoprotein antibody-related demyelinating diseases comprising optic neuritis, neuromyelitis optica spectrum disorder, and ADEM $[15,16]$. Although previous investigations largely focused on how B cells contribute to the pathogenesis of MS, similar implications can be made in other inflammatory demyelinating CNS diseases such as ADEM [17]. On this basis, rituximab can be considered as a treatment option even in EBV-negative ADEM patients who failed to show improvement with first-line immunotherapy. Further research is warranted in this area.

Of note, although rituximab has been proven to be safe, it can increase the risk of infectious complications, including hepatitis $B$ virus, progressive multifocal leukoencephalopathy, enterovirus encephalitis, parvovirus B19, and cytomegalovirus [18]. Clinicians should take caution, especially in cases of advanced immune compromise, and patients should be monitored closely for complications. Screening for evidence of hepatitis B in all patients and appropriate preventive measures or treatment should be implemented before initiating rituximab therapy. 


\section{Conflicts of Interest}

Kon Chu has been on an editorial board for encephalitis since October 2020. He was not involved in the review process of this case report. No other potential conflict of interest relevant to this article was reported.

\section{Author's Contributions}

Data curation: all authors; Writing-original draft: Kim S; Writing-review and editing: Ahn SJ, Chu K.

\section{References}

1. Pohl D, Alper G, Van Haren K, et al. Acute disseminated encephalomyelitis: updates on an inflammatory CNS syndrome. Neurology 2016; 87(9 Suppl 2):S38-S45.

2. Z Ghali MG. Tumefactive acute disseminated encephalomyelitis. Neurol India 2020;68:35-41.

3. Triulzi F. Neuroradiology of multiple sclerosis in children. Neurol Sci 2004;25 Suppl 4:S340-S343.

4. Koelman DL, Chahin S, Mar SS, et al. Acute disseminated encephalomyelitis in 228 patients: a retrospective, multicenter US study. Neurology 2016;86:2085-2093.

5. Menge T, Hemmer B, Nessler S, et al. Acute disseminated encephalomyelitis: an update. Arch Neurol 2005;62:1673-1680.

6. Wender M. Acute disseminated encephalomyelitis (ADEM). J Neuroimmunol 2011;231:92-99.

7. Hatton OL, Harris-Arnold A, Schaffert S, Krams SM, Martinez OM. The interplay between Epstein-Barr virus and B lymphocytes: implications for infection, immunity, and disease. Immunol Res 2014;58:268-276.

8. Fujimoto H, Asaoka K, Imaizumi T, Ayabe M, Shoji H, Kaji M. Epstein-Barr virus infections of the central nervous system. Intern Med
2003;42:33-40.

9. Bahadori HR, Williams VC, Turner RP, et al. Acute disseminated encephalomyelitis following infectious mononucleosis. J Child Neurol 2007;22:324-328.

10. Shoji H, Kusuhara T, Honda Y, et al. Relapsing acute disseminated encephalomyelitis associated with chronic Epstein-Barr virus infection: MRI findings. Neuroradiology 1992;34:340-342.

11. Paskavitz JF, Anderson CA, Filley CM, Kleinschmidt-DeMasters BK, Tyler KL. Acute arcuate fiber demyelinating encephalopathy following Epstein-Barr virus infection. Ann Neurol 1995;38:127-131.

12. Nakamura Y, Nakajima H, Tani H, et al. Anti-MOG antibody-positive ADEM following infectious mononucleosis due to a primary EBV infection: a case report. BMC Neurol 2017;17:76.

13. Ali D, Cardos B, Gorur Y, et al. A rare case of adult acute disseminated encephalomyelitis associated with primary Epstein-Barr virus infection. Eur J Case Rep Intern Med 2019;6:001094.

14. Oh H, Yoo JR, Heo S, Oh JH, Lee HK. Acute disseminated encephalomyelitis caused by Epstein-Barr virus infection in an immunocompetent adult woman. J Med Life Sci 2019;16:17-22.

15. Armangue T, Olivé-Cirera G, Martínez-Hernandez E, et al. Associations of paediatric demyelinating and encephalitic syndromes with myelin oligodendrocyte glycoprotein antibodies: a multicentre observational study. Lancet Neurol 2020;19:234-246.

16. Ramanathan S, Mohammad S, Tantsis E, et al. Clinical course, therapeutic responses and outcomes in relapsing MOG antibody-associated demyelination. J Neurol Neurosurg Psychiatry 2018;89:127-137.

17. McLaughlin KA, Wucherpfennig KW. B cells and autoantibodies in the pathogenesis of multiple sclerosis and related inflammatory demyelinating diseases. Adv Immunol 2008;98:121-149.

18. Gea-Banacloche JC. Rituximab-associated infections. Semin Hematol 2010;47:187-198. 